\title{
First-line pembrolizumab
} plus chemotherapy for extensive-stage small-cell lung cancer: a United States-based
cost-effectiveness analysis

\author{
Youwen Zhu' ${ }^{1}$, Huabin $\mathrm{Hu}^{2,3}$, Dong Ding ${ }^{1}$, Shuosha $\mathrm{Li}^{1}$, Mengting Liao ${ }^{4}$, Yin Shi ${ }^{5}$ and Jin Huang ${ }^{1,6^{*}}$ (I)
}

\begin{abstract}
Background: The clinical trial of Keynote-604 showed that pembrolizumab plus chemotherapy could generate clinical benefits for extensive-stage small-cell lung cancer (ES-SCLC). We aim to assess the efficacy and cost of pembrolizumab combined with chemotherapy in the first-line treatment setting of ES-SCLC from the United States (US) payers' perspective.

Methods: A synthetical Markov model was used to evaluate cost and effectiveness of pembrolizumab plus platinum-etoposide(EP) versus EP in first-line therapy for ES-SCLC from the data of Keynote-604. Lifetime costs lifeyears(LYs), quality adjusted LYs(QALYs) and incremental cost-effectiveness ratios(ICERs) were estimated. One-way and probabilistic sensitivity analyses were performed. Furthermore, we performed subgroup analysis.

Results: Pembrolizumab plus EP resulted in additional $0.18 \mathrm{QALYS}(0.32 \mathrm{LYS})$ and corresponding incremental costs $\$ 113,625$, resulting an ICER of $\$ 647,509$ per QALY versus EP. The price of pembrolizumab had a significant impact on ICER. Probabilistic sensitivity analysis indicated that pembrolizumab combined chemotherapy may become a cost-effective option with a probability of 0\%. Besides, subgroup analysis suggested that all subgroups were not cost-effective.
\end{abstract}

Conclusion: From the perspective of the US payer, pembrolizumab plus EP is not a cost-effective option for first-line treatment patients with ES-SCLC at a WTP threshold of $\$ 150,000$ per QALY.

Keywords: Pembrolizumab, ES-SCLC, Platinum-etoposide, Cost-effectiveness, Quality-adjusted life-years

\section{Background}

Lung cancer is the main cause of cancer-related death in the United States (US), accounting for more than 13\% incidence and 22\% mortality in 2021 [1]. Small-cell lung cancer (SCLC) accounts for $10-15 \%$ of lung cancers and has a high rate of early metastasis (up to $60-70 \%$ ) [2, 3]. Overall survival (OS) depends on the initial stage of

*Correspondence: jinhuang@csu.edu.cn

1 Department of Oncology, Xiangya Hospital, Central South University, Changsha 410008, Hunan, China

Full list of author information is available at the end of the article diagnosis, with a 5 -year survival rate of $20-25 \%$ for localized-stage SCLC (LS-SCLC) and only $2 \%$ for extensivestage small cell lung cancer (ES-SCLC) $[4,5]$.

The emergence of immune checkpoint inhibitors (ICIs) has broken the dominant position of etoposide and platinum (EP) regimen in the first-line treatment of ES-SCLC for more than thirty years. ICIs include anti-programmed cell death protein 1 (PD-1) and anti-PD-1 Ligand antibodies (PD-L1), against cytotoxic T-lymphocyte antigen 4 (CTLA-4), some of which have been approved for clinical use $[6,7]$. 
Pembrolizumab is a selective, high-affinity, programmatic, specific, human immunoglobulin G4 monoclonal antibody that binds to the programmed death 1 receptor. It allows anti-tumor $\mathrm{T}$ cells to recognize and kill tumor cells [8]. After platinum-based chemotherapy and other therapies failed for advanced SCLC, pembrolizumab monotherapy was approved by the US Food and Drug Administration (FDA) in 2019 [9]. In the first line treatment of patients with ES-SCLC, the randomized phase 3 trial Keynote-604 further demonstrated the efficacy of the combination of pembrolizumab with chemotherapy. It showed that pembrolizumab plus EP therapy prolonged overall survival (OS 10.8 vs 9.7 months; hazard ratio [HR] $0.8 ; 95 \% \mathrm{CI} 0.64$ to $0.98 ; \mathrm{p}=0.0164)$ and significantly extended the progression-free survival (PFS 4.5 vs 4.3 months; HR 0.75 ; $95 \%$ CI 0.61 to $0.91 ; \mathrm{p}=0.0023$ ) compared to EP therapy [10].

However, considering its high cost and limited potential population, economic analysis is urgently needed to evaluate whether a newly approved therapy provides clinical benefit at a justifiable cost and is increasingly necessary to expand its application. Therefore, our goal was to estimate the cost-effectiveness of pembrolizumab plus EP as the first-line treatment of ES-SCLC from the US payers' perspective.

\section{Methods}

\section{Model structure}

A synthetical Markov model combining a decision tree was established to assess the costs and effectiveness of the different first-line treatment of ES-SCLC for patients, and was similar to the Keynote-604 trial. The decision trees included two treatment: pembrolizumab plus EP group and EP group. The Markov model included three health states to represent the disease course of ES-SCLC: PFS, progressive disease (PD), and death (Additional file 1: Fig. S1 in the electronic supplementary material). All patients in the model started from the PFS state and were treated with pembrolizumab plus EP or EP until disease progression or intolerable toxicity and adverse effects. After PD, patients could receive subsequent treatment, with death as the terminal state. The Markov cycle length in the model was 6 weeks and outcomes were runs for 10 years boundaries. We set the costs and effects of a 3\% discount rate per year [11]. Costs, LYs, QALYs, and calculated ICERs were estimated in each treatment regimen. We also considered sub-group cost-effectiveness analysis. The model structure and data were based on results of Keynote-604 [10], the US publicly available databases and published literature [12-15]. The model was constructed viaTreeAge Pro 2020 software (TreeAge Software, Williamstown, MA, https://www.treeage.com).

\section{Model survival and progression risk estimates}

The model operated transition probabilities between health states derived from PFS, OS, and death curves from Keynote-604. We applied the GetData Graph Digitizer (version 2.26; http://www.getdata-graph-digitizer. com/index.php) to collect the data points from the OS and PFS curves and these data points were then used to fit parametric survival models. Then, many parametric survival models were fitted to time-to-event data extracted from Keynote-604 trial, including the Weibull, Exponential, Gompertz, Log-logistic, and Log-normal distributions. According to all aspects, Akaike's information criterion (AIC) and Bayesian information criterion (BIC) are selected to evaluate the fitting degree of the alternative model. Weibull survival curves which were flexible and widely used were matched to the number of patients in the three states over time, as it can monotonically increase or decrease the hazard function, it is suitable for estimating the event that occurs in the early follow-up work period. The survival model selection was shown in previously published research [16]. the survival model selection was shown in Additional file 1: Table S3 and Fig. S2. Then, we used Weibull distribution to operate in R and we got the two parameters, shape $(\gamma)$ and scale $(\lambda)$, were estimated from this fit and applied to Kaplan-Meier curves using the $\mathrm{R}$ (version 4.0.2, http:// www.r-project.org) and the method proposed by Hoyle and Henley [17].

The time-dependency transition probabilities (tp) are essential in the model analysis. Tp in each Markov cycle was calculated based on the following formula: The Markov cycle is $\mathrm{u}$ and the arrival at state $\mathrm{t}$ after $\mathrm{u}$ Markov cycles is tu was calculated with the following formula: $\operatorname{tp}(\mathrm{tu})=1-\exp \{\lambda(\mathrm{t}-\mathrm{u}) \gamma-\lambda \mathrm{t} \gamma\}(\lambda>0, \gamma>0)[18]$.

\section{Utility estimates}

Utility was used to estimate consumer's quality of life (QoL) in the natural history of the disease, on a scale of 0 (death) to 1 (health). We considered the mean health utility score of 0.840 [12] and 0.473 [13] for the PFS and the PD state, respectively. They were based on previously published articles. We also consider the disutility values of $3 / 4$ adverse events (AEs) in our analysis [13-15].

\section{Cost inputs}

We only considered direct costs in 2021 US dollars as follows: the costs of medicines [19, 20], AEs costs (assuming that AEs appeared only one cycle in the PFS and the PD state) [21, 22], costs of laboratory [23] and imaging (every 6 weeks for the first 48 weeks, and every 9 weeks thereafter) [24] and tests administration costs [25] (Table 1 and Additional file 1: Table S1). Based on 
Table 1 Model parameters: baseline values, ranges, and distributions for sensitivity analysis

\begin{tabular}{|c|c|c|c|c|c|}
\hline \multirow[t]{2}{*}{ Variable } & \multirow[t]{2}{*}{ Baseline value } & \multicolumn{2}{|l|}{ Range } & \multirow[t]{2}{*}{ Reference } & \multirow[t]{2}{*}{ Distribution } \\
\hline & & Minimum & Maximum & & \\
\hline $\begin{array}{l}\text { Weibull survival model of OS of PEP } \\
\text { Weibull survival model of OS of EP }\end{array}$ & $\begin{array}{l}\text { Scale }=0.035532 \\
\text { Shape }=1.209467 \\
\text { Scale }=0.021012 \\
\text { Shape }=1.497332\end{array}$ & - & $\begin{array}{l}- \\
-\end{array}$ & [10] & - \\
\hline $\begin{array}{l}\text { Weibull survival model of PFS of PEP } \\
\text { Weibull survival model of PFS of EP }\end{array}$ & $\begin{array}{l}\text { Scale }=0.06893 \\
\text { Shape }=1.40287 \\
\text { Scale }=0.02532 \\
\text { Shape }=2.21179\end{array}$ & - & $\begin{array}{l}- \\
-\end{array}$ & [10] & $\begin{array}{l}- \\
-\end{array}$ \\
\hline \multicolumn{6}{|c|}{ Risk for main adverse events in PEP group } \\
\hline Risk of neutropenia & 0.435 & 0.348 & 0.522 & [10] & Beta \\
\hline Risk of anemia & 0.157 & 0.126 & 0.189 & [10] & Beta \\
\hline Risk of thrombocytopenia & 0.139 & 0.111 & 0.167 & [10] & Beta \\
\hline Risk of leucopenia & 0.117 & 0.094 & 0.140 & [10] & Beta \\
\hline Risk of pneumonia & 0.067 & 0.054 & 0.080 & {$[10]$} & Beta \\
\hline \multicolumn{6}{|l|}{ Risk for main adverse events in EP group } \\
\hline Risk of neutropenia & 0.408 & 0.326 & 0.490 & [10] & Beta \\
\hline Risk of anemia & 0.152 & 0.121 & 0.182 & [10] & Beta \\
\hline Risk of thrombocytopenia & 0.112 & 0.090 & 0.134 & [10] & Beta \\
\hline Risk of leucopenia & 0.094 & 0.075 & 0.113 & [10] & Beta \\
\hline \multicolumn{6}{|l|}{ Utility } \\
\hline Utility PFS in first-line treatment & 0.840 & 0.672 & 1.008 & [12] & Beta \\
\hline Utility PD & 0.473 & 0.378 & 0.568 & [13] & Beta \\
\hline \multicolumn{6}{|l|}{ Disutility due to AEs } \\
\hline Neutropenia & 0.09 & 0.072 & 0.108 & [13] & Beta \\
\hline Anemia & 0.073 & 0.058 & 0.088 & [13] & Beta \\
\hline Leucopenia & 0.09 & 0.072 & 0.108 & [13] & Beta \\
\hline Pneumonia & 0.09 & 0.072 & 0.108 & [14] & Beta \\
\hline Thrombocytopenia & 0.02 & 0.016 & 0.024 & [15] & Beta \\
\hline \multicolumn{6}{|l|}{ Drug cost, \$/per mg } \\
\hline Pembrolizumab & $20,370.80$ & $16,296.64$ & $24,444.96 \square$ & {$[20]$} & Gamma \\
\hline Etoposide & $1,663.73$ & $1,330.98$ & 1996.47 & [20] & Gamma \\
\hline Carboplatin & 85.83 & 68.66 & 103.00 & [20] & Gamma \\
\hline Cisplatin & 51.78 & 41.42 & 62.13 & [20] & Gamma \\
\hline Topotecan & 199.87 & 159.90 & 239.84 & [20] & Gamma \\
\hline \multicolumn{6}{|l|}{ Expenditures on main adverse events, \$ } \\
\hline Leucopenia & $6,831.97$ & $5,465.58$ & $8,198.37$ & [21] & Gamma \\
\hline Neutropenia & 733.36 & 586.69 & 880.03 & [22] & Gamma \\
\hline Anemia & $1,199.86$ & 959.89 & $1,439.83$ & [22] & Gamma \\
\hline Thrombocytopenia & 851.51 & 681.21 & $1,021.82$ & [22] & Gamma \\
\hline Pneumonia & $5,646.88$ & $4,517.51$ & $6,776.26$ & [22] & Gamma \\
\hline Laboratory per cycle & 315.14 & 252.11 & 378.17 & [23] & Gamma \\
\hline Tumor imaging per cycle & 231.10 & 184.88 & 167.60 & [24] & Gamma \\
\hline Administration per cycle & 139.67 & 111.74 & 167.60 & {$[25]$} & Gamma \\
\hline Discount rate & 0.03 & - & - & [11] & - \\
\hline
\end{tabular}

HR: hazard ratio; OS: overall survival; PFS: progression-free survival; EP: Etoposide and Platinum; PEP: pembrolizumab plus Etoposide and Platinum; AEs: adverse events 
the Keynote-604 trial, the patients in pembrolizumab plus chemotherapy group: pembrolizumab at a dose of $200 \mathrm{mg}$ once every 3 weeks for 35 cycles and EP [etoposide at a dose of $100 \mathrm{mg} / \mathrm{m}^{2}$ on days 1,2 , and 3 and the investigator's choice of carboplatin (71.10\%) at an area under the curve $5 \mathrm{mg} / \mathrm{mL}$ per min or cisplatin (28.9\%) at a dose of $75 \mathrm{mg} / \mathrm{m}^{2}$ every 3 weeks]. The patients in chemotherapy group: EP every 3 weeks for 4 cycles. Patients in the pablizumab plus chemotherapy group received four cycles of induction therapy followed by intravenous injection of $200 \mathrm{mg}$ pablizumab as maintenance therapy. $16.6 \%$ patients in the pembrolizumab plus chemotherapy group and $21.1 \%$ patients in the chemotherapy group received subsequent anticancer therapy.

The median dosage of medicines was estimated based on standard patients: area under the concentration curve of $5 \mathrm{mg} / \mathrm{mL} / \mathrm{min}$ and assumed serum creatinine of 1 , male sex, 65 years of age, weight of $70 \mathrm{~kg}$, height of 70 inches, and body surface area $1.84 \mathrm{~m}^{2}$ [26]. Grade $1 / 2$ events were considered manageable within standard patient monitoring and the correlation with QoL was low [27]. Thus, we included only the cost of managing grade $3 / 4$ AEs (a frequency of greater than 5\%) in the model, which had notably different probabilities between the arms of the Keynot-604 trial. All relevant parameters were shown in Table 1.

\section{Sensitivity analysis}

We used a series of sensitivity analyses to predict the uncertainty of the model results. One-way sensitivity analysis was conducted within a variance of $20 \%$ from their baseline values according to varied values of a certain parameter within its defined range and the established approaches to examined the individual effects of this parameter on the ICERs (Table 1) [11, 28]. We also conducted probabilistic sensitivity analyses by performing 10,000 Monte Carlo simulations, and the probabilistic sensitivity analysis was completed to assess the variations in multiple parameters at once [29]. A cost-effectiveness acceptability curve of each treatment strategy was evaluated as being the most cost-effective at a certain WTP threshold.

We also considered all patient subgroups of the Keynote-604 trial. In the absence of sufficient data for each patient subgroup, the study adopted the same baseline chemotherapy survival curve for all patients in the chemotherapy group, and their pembrolizumab plus chemotherapy survival curves were produced based on the subgroup-specific HRs according to the approach taken by Hoyle et al. [30] for the absence of OS and PFS curves for each patient subgroup.

\section{Results}

Base case results

The model projected that life expectancy of patients receiving pembrolizumab plus EP was 1.83 LYs, which was $0.32 \mathrm{LYs}$ more than patients receiving EP. Accounting for QOL, patients receiving pembrolizumab plus EP gained 1.07 QALYs; this value was 0.18 QALYs more than for patients receiving EP. The use of pembrolizumab plus EP cost an additional $\$ 113,625$, resulting in an ICER of $\$ 346,818$ per LY, or $\$ 647,509$ per QALY compared with EP (Table 2).

\section{Sensitivity analysis}

One-way sensitivity analysis results (Fig. 1) were most sensitive to the changes in the price of pembrolizumab(ranging from $\$ 40.74$ to $\$ 61.11$ per $\mathrm{mg}$, with the ICER increasing from $\$ 523,581$ per QALY to $\$ 771,438$ per QALY), followed by the utility of PD, the utility of PFS, the risk of neutropenia in pembrolizumab plus chemotherapy group, the risk of neutropenia in pembrolizumab plus chemotherapy group, and the risk of leucopenia in pembrolizumab plus chemotherapy group.

The ICER scatter diagram (Additional file 1: Fig. S3) showed that the probability sensitivity analysis and pembrolizumab plus EP cannot be effective at the WTP threshold of \$150,000 per QALY.

As shown in the cost-effectiveness acceptability curve(Fig. 2), the probability that the pembrolizumab plus EP strategy is cost-effective increases as the WTP for additional QALY rises. When the cost of pembrolizumab was reduced by $80.3 \%$, and the ICER was $\$ 149,904 /$ QALY, which pembrolizumab plus EP was cost-effective. Subgroup analysis declared that the ICER of all patient subgroups was still greater than $\$ 150,000$ / QALY(Additional file 1: Table S2).

Table 2 Baseline results

\begin{tabular}{|c|c|c|}
\hline Parameters & Pembrolizumab plus EP & EP \\
\hline LYS & 1.83 & 1.51 \\
\hline QALYS & 1.07 & 0.89 \\
\hline Total cost \$ & 130,692 & 17,067 \\
\hline ICER \$/LY a & 346,818 & - \\
\hline ICER \$/QALY $b$ & 647,509 & - \\
\hline WTP \$/QALY & 150,000 & - \\
\hline
\end{tabular}

EP: Etoposide and Platinum; ICER: incremental cost-effectiveness ratio; LY: lifeyear; QALY: quality-adjusted life-year; WTP: willingness-to-pay

a Compared to EP $(\$ / L Y)$

${ }^{b}$ Compared to EP (\$/QALY) 


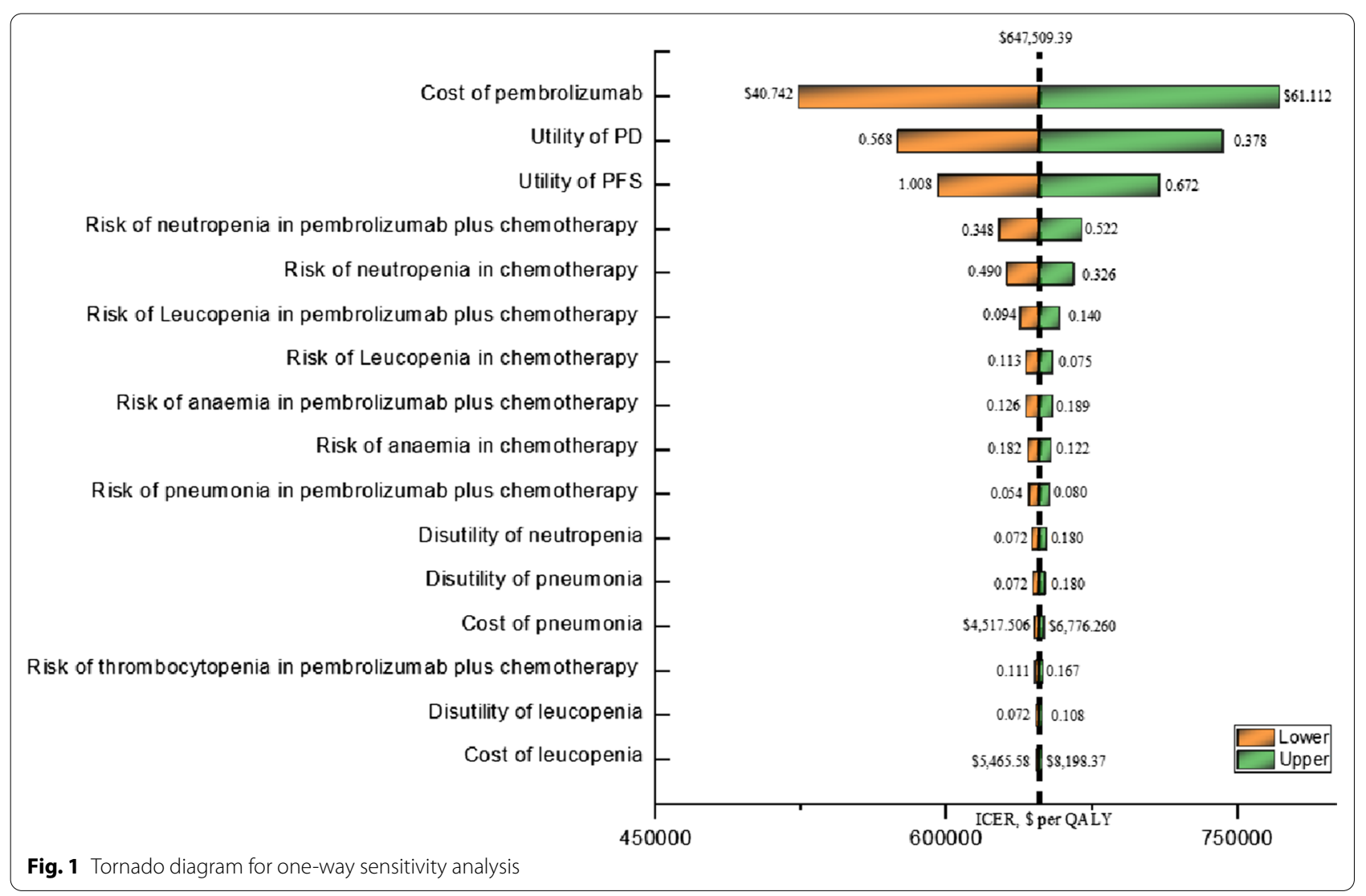

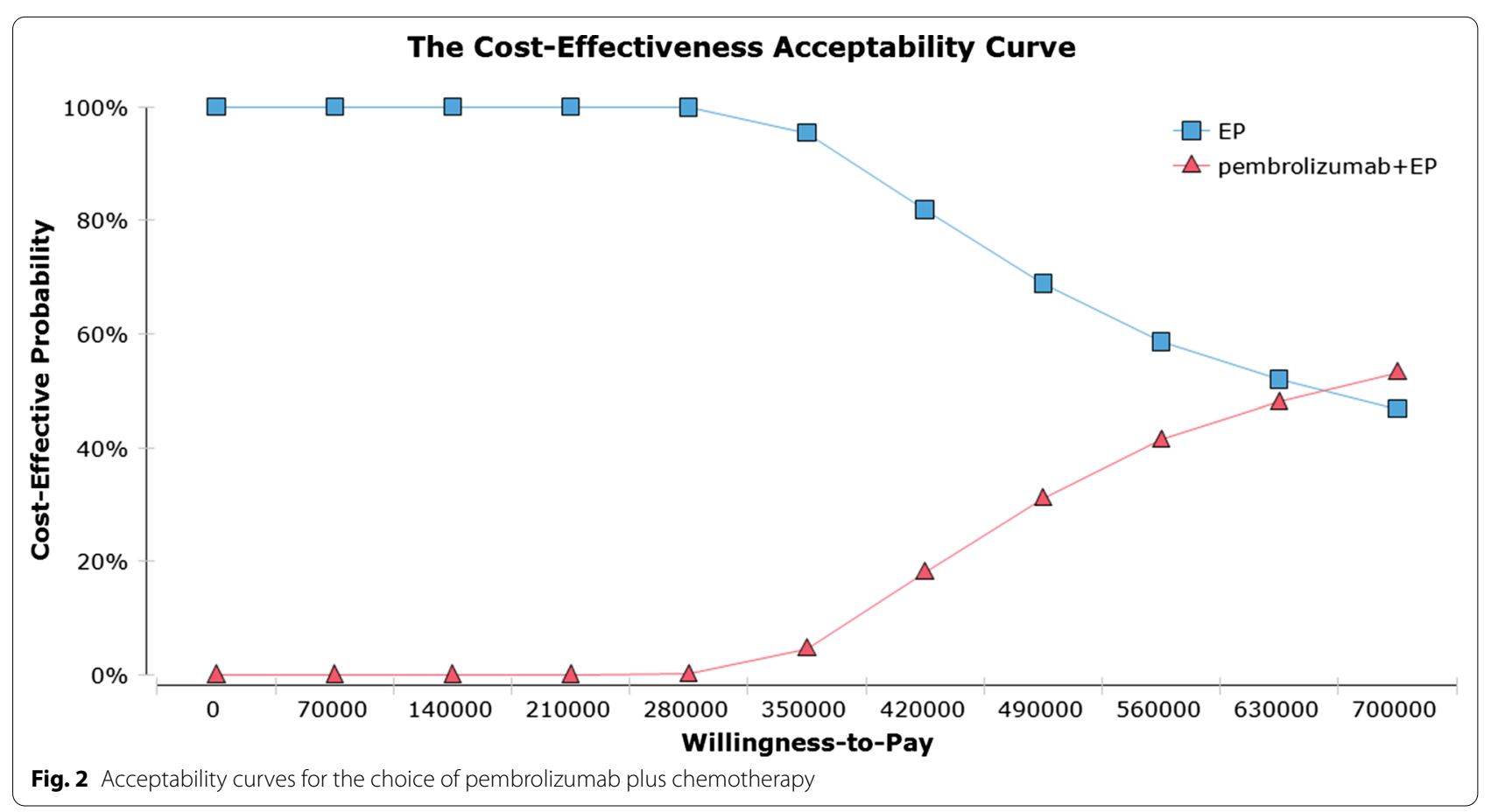




\section{Discussion}

In recent years, the emergence of ICIs has greatly changed the treatment strategy of lung cancer, raising a great interest in oncologists and patients. There were two studies evaluated the efficacy of ICIs in the firstline treatment of ES-SCLC, Both in CASPIAN [31] and Impower133 [32] trials, first-line immunotherapy plus chemotherapy showed satisfied efficacy. Based on these clinical trials, published research studied the cost-effectiveness of new immunotherapy strategies. According to the data of CASPIAN trial, our published research [33] demonstrated that durvalumab in combination with platinum-etoposide was not a cost-effective option in the first-line treatment of patients with extensive-stage SCLC in the U.S. According to the data of Impower133 trial, Qiu Li [34] and Li [15] conducted cost-effectiveness analysis and concluded that atezolizumab plus chemotherapy to chemotherapy was not a cost-effective choice in the first-line treatment of extensive-stage SCLC from an American and Chinese perspective, respectively. The common point of these studies is that the price of PD-L1 antibody was always the greatest factor affecting the outcomes. Given the updated survival data of the Keynote-604 trial recently published, the cost-effectiveness of pembrolizumab plus chemotherapy in the first-line treatment for patients with ES-SCLC is necessary to be updated accordingly. Therefore, we performed the first cost-effectiveness analysis of pembrolizumab plus chemotherapy versus chemotherapy in first-line setting for patients with ES-SCLC.

Our analysis proved that pembrolizumab plus chemotherapy was not cost-effective in the first-line treatment of ES-SCLC at a WTP threshold of \$150,000 per QALY, resulting in an additional 0.18 QALYs and ICER of $\$$ $\$ 647,509$ per QALY versus chemotherapy. One-way sensitivity analysis showed that the cost of pembrolizumab was the most influential factor. Further, analysis found that when the cost of pembrolizumab was reduced by $80.3 \%$, the immunotherapy became cost-effective at an ICER of $\$ 149,904 / \mathrm{QALY}$. Therefore, changing the price of pembrolizumab is an effective feasible strategy to achieve efficient use of pembrolizumab plus chemotherapy. The acceptability curves also demonstrated this finding that a paucity of certainty was achieved by pembrolizumab plus chemotherapy at the WTP threshold of $\$ 150,000$ in the US. The results of probabilistic sensitivity analysis showed that the probability of pembrolizumab plus chemotherapy was cost-effective vs chemotherapy was $0 \%$. The subgroup cost-effectiveness analysis demonstrated that pembrolizumab plus chemotherapy was not a cost-effective treatment across all patients' subgroups. Our analysis will be essential to guide policymaking and payment in health care and provided drug pricing decision-makers with the reference to reprice pembrolizumab.

Unfortunately, the detailed expression of PD-L1 and tumor mutational burden (TMB) were not shown in the Keynote-604 trial. In many other studies, PD-L1 expression and TMB were of great value as biomarkers for the efficacy of ICIs treatment, which can improve clinical benefits [35-42]. Anti-PD-1/PD-L1 antibody is effective in patients with high expression of PD-L1 and high TMB (TMB-H), especially in patients with non-small cell lung cancer (NSCLC), melanoma, renal cell carcinoma, etc. $[43,44]$. In our previous studies, we showed that first-line treatment with pembrolizumab was a cost-effective strategy when compared to platinum-based chemotherapy in locally advanced or metastatic NSCLC patients with high expression of PD-L1 (TPS $\geq 50 \%$ ) [45]. Regardless of the PD-L1 expression levels nivolumab plus ipilimumab was cost-effective in NSCLC patients with TMB-H [46]. It seems that the significance of using predictive markers to properly screen patients and achieve cost-effective strategies is a concern of clinicians and administrators [47]. Therefore, more detailed information about biomarkers is needed in future studies, including PD-L1 expression, TMB, microsatellite instability (MSI) and deficient DNA mismatch repair (dMMR), etc.

Significantly, despite the survival benefits displayed in certain clinical trials, ICIs were not cost-effective in most cases. Consequently, it is a great challenge that to approve new drugs solely based on cost-effectiveness of drugs without considering the dynamic evolution of survival curves and using predictive markers.

There are some limitations in our study. Firstly, our study is based on the Keynote-604 trial, the only clinical trial that estimated the efficacy of pembrolizumab plus EP or EP as first-line therapy in patients with ES-SCLC. Any bias within the trial will be reflected in our study. Secondly, Some of the utility values used in our research are not based on the US, which is indeed an inevitable error. Third,the long-term efficacy of pembrolizumab plus EP in the model was extrapolated from the clinical data from the Keynote-604 trial, which is inevitably subject to uncertainty. Fourth, the Keynote-604 trial did not provide the Kaplan-Meier curve for each subgroup, making it impossible to run the model completely for each subgroup. The original group balance produced by randomization may not exist in the subgroups. Thus, the results of the subgroup analyses should be interpreted with caution. Fifth, we revised the utility value by the disutility of AEs, which will lead to the inaccuracy of the utility value. Sixth, the costs of grade $1 / 2$ AEs and immune-related AEs were excluded, which might overestimate the benefits of pembrolizumab plus chemotherapy. Finally, the compliance of patients was not 
considered in our study, while a large number of studies have shown that compliance has a significant impact on cost-effectiveness for cancer patients.

\section{Conclusion}

From the perspective of the US payer, pembrolizumab plus EP is not a cost-effective option for first-line treatment patients with ES-SCLC at a WTP threshold of $\$ 150,000$ per QALY. This finding may help decisionmaking in healthcare and policy formulation in medical reimbursement.

\section{Supplementary Information}

The online version contains supplementary material available at https://doi. org/10.1186/s12962-021-00329-w.

Additional file 1: Table S1. Drug dose and costs. Table S2. Results of subgroup analyses. Table S3. Summary of statistical goodness-of-fit of K-M curve in Keynote-604 trial. Figure S1. Markov states. Figure S2. Kaplan-Meier Curve Fitting and Extrapolation. Figure S3. Probability Sensitivity Analysis Scatter Plot.

\section{Acknowledgements}

This manuscript was supported by grants from the Hunan Natural Science Foundation of China (No. 2018JJ3852).

\section{Authors' contributions}

Performed the experiments: YWZ, DD, HHB, SSL. Analyzed the data: YWZ, $\mathrm{DD}, \mathrm{HHB}, \mathrm{SSL}, \mathrm{YS}, \mathrm{MTL}, J \mathrm{H}$. Contributed reagents/materials/analysis tools: JH. Wrote the manuscript:YWZ DD HHB JH. All authors read and approved the manuscript.

\section{Funding}

This manuscript was supported by grants from the Hunan Natural Science Foundation of China (No. 2018JJ3852); The authors have no other relevant affiliations or financial involvement with any organization or entity with a financial interest in or financial conflict with the subject matter or materials discussed in the manuscript apart from those disclosed.

\section{Availability of data and materials}

All authors had full access to all of the data in this study and take complete responsibility for the integrity of the data and accuracy of the data analysis. The datasets generated and/or analyzed during the current study are available from the corresponding author on reasonable request.

\section{Declarations}

\section{Ethics approval and Consent to Participate}

Not applicable.

\section{Competing interests}

All of the authors have indicated that they have no competing interests with regard to the content of article. This manuscript is original and has not been previously published, nor has it been simultaneously submitted to any other journal.

\section{Author details}

${ }^{1}$ Department of Oncology, Xiangya Hospital, Central South University, Changsha 410008, Hunan, China. '2Department of Medical Oncology, The Sixth Affiliated Hospital of Sun Yat-Sen University, Guangzhou 510655, China. ${ }^{3}$ Guangdong Institute of Gastroenterology, Guangdong Provincial Key Laboratory of Colorectal and Pelvic Floor Diseases, Guangzhou 510655 China. ${ }^{4}$ Department of Health Management Center, Xiangya Hospital, Central South University, Changsha 410008, Hunan, China. ${ }^{5}$ Department of Pharmacy,
Xiangya Hospital, Central South University, Changsha 410008, Hunan, China. ${ }^{6}$ Department of Dermatology, Hunan Engineering Research Center of Skin Health and Disease, Hunan Key Laboratory of Skin Cancer and Psoriasis, Xiangya Hospital, Central South University, Changsha 410008, Hunan, China.

Received: 20 July 2021 Accepted: 30 October 2021

Published online: 04 December 2021

\section{References}

1. Siegel RL, Miller KD, Fuchs HE, Jemal A. Cancer statistics, 2021. CA Cancer J Clin. 2021;71:7-33.

2. Nicholson AG, Chansky K, Crowley J, et al. The International Association for the Study of Lung Cancer Lung Cancer Staging Project: proposals for the revision of the clinical and pathologic staging of small cell lung cancer in the forthcoming eighth edition of the TNM classification for lung cancer. J Thorac Oncol. 2016;11:300-11.

3. Rudin CM, Giaccone G, Ismaila N. Treatment of small-cell lung cancer: American society of clinical oncology endorsement of the american college of chest physicians guideline. J Oncol Pract. 2016;12:83-6.

4. Tsiouprou I, Zaharias A, Spyratos D. the role of immunotherapy in extensive stage small-cell lung cancer: a review of the literature. Can Respir J. 2019;2019:6860432.

5. Zimmerman S, Das A, Wang S, et al. 2017-2018 Scientific advances in thoracic oncology: small cell lung cancer. J Thorac Oncol. 2019;14:768-83.

6. Yang S, Zhang Z, Wang Q. Emerging therapies for small cell lung cancer. J Hematol Oncol. 2019:12:47.

7. Farago AF, Keane FK. Current standards for clinical management of small cell lung cancer. Transl Lung Cancer Res. 2018;7:69-79.

8. Kwok G, Yau TCC, Chiu JW, et al. Pembrolizumab (Keytruda). Hum Vaccin Immunother. 2016;12:2777-89.

9. US Food and Drug Administration.FDA approves pembrolizumab for metastatic small cell lung cancer.FDA.2019. https://www.fda.gov/drugs/ resources-information-approved-drugs/fda-approves-pembrolizumabmetastatic-small-cell-lung-cancer Accessed 18 Jun 2019.

10. Rudin CM, Awad MM, Navarro A, et al. Pembrolizumab or placebo plus etoposide and platinum as first-line therapy for extensive-stage small-cell lung cancer: randomized, double-blind, phase III KEYNOTE-604 Study. J Clin Oncol. 2020;38:2369-79.

11. Goldstein DA, Chen Q, Ayer T, et al. First- and second-line bevacizumab in addition to chemotherapy for metastatic colorectal cancer: a United States-based cost-effectiveness analysis. J Clin Oncol. 2015;33:1112-8.

12. Yang S-C, Kuo C-W, Lai W-W, et al. Dynamic changes of health utility in lung cancer patients receiving different treatments: a 7-year follow-up. J Thorac Oncol. 2019;14:1892-900.

13. Nafees B, Stafford M, Gavriel S, et al. Health state utilities for non small cell lung cancer. Health Qual Life Outcomes. 2008;6:84

14. Zeng X, Wan X, Peng $L$, et al. Cost-effectiveness analysis of pembrolizumab plus chemotherapy for previously untreated metastatic non-small cell lung cancer in the USA. BMJ Open. 2019;9:e031019.

15. Li L-Y, Wang H, Chen $X$, et al. First-line atezolizumab plus chemotherapy in treatment of extensive small cell lung cancer. Chin Med J. 2019;132:2790-4

16. Latimer NR. Survival analysis for economic evaluations alongside clinical trials - extrapolation with patient-level data. Med Decis Mak. 2013:33:743-54.

17. Hoyle MW, Henley W. Improved curve fits to summary survival data: application to economic evaluation of health technologies. BMC Medical Research Methodology. 2011;11:139.

18. Zeng X, Li J, Peng L, et al. Economic outcomes of maintenance gefitinib for locally advanced/metastatic non-small-cell lung cancer with unknown EGFR mutations: a semi-Markov model analysis. PLoS ONE. 2014:9:e88881.

19. https://www.cms.gov/medicare/medicare-part-b-drug-average-salesprice/2021-asp-drug-pricing-files. Accessed April 2021

20. https://www.drugs.com/price-guide/ Accessed October,2020.

21. Rashid N, Koh H, Baca H, et al. Economic burden related to chemotherapy-related adverse events in patients with metastatic breast cancer in an integrated health care system. Breast Cancer: Targets Ther. 2016;8:173-81. 
22. Insinga RP, Vanness DJ, Feliciano $J$, et al. Cost-effectiveness of pembrolizumab in combination with chemotherapy versus chemotherapy and pembrolizumab monotherapy in the first-line treatment of squamous non-small-cell lung cancer in the US. Curr Med Res Opin. 2019;35:1241-56.

23. Mistry R, May JR, Suri G, et al. Cost-effectiveness of ribociclib plus letrozole versus palbociclib plus letrozole and letrozole monotherapy in the firstline treatment of postmenopausal women with HR+/HER2 — advanced or metastatic breast cancer: a U.S. payer perspective. J Manag Care Spec Pharm. 2018;24:514-23.

24. Carlson JJ, Canestaro W, Ravelo A, Wong W. The cost-effectiveness of alectinib in anaplastic lymphoma kinase-positive (ALK+) advanced NSCLC previously treated with crizotinib. J Med Econ. 2017;20:671-7.

25. Wan X, Zhang Y, Tan C, et al. First-line nivolumab plus ipilimumab vs sunitinib for metastatic renal cell carcinoma. JAMA Oncol. 2019;5:491-6.

26. Handorf EA, McElligott S, Vachani A, et al. Cost effectiveness of personalized therapy for first-line treatment of stage IV and recurrent incurable adenocarcinoma of the lung. J Oncol Pract. 2012;8:267-74.

27. de Groot S, Redekop WK, Versteegh MM, et al. Health-related quality of life and its determinants in patients with metastatic renal cell carcinoma. Qual Life Res. 2018;27:115-24.

28. Zhang Y, Baik SH, Fendrick AM, Baicker K. Comparing local and regional variation in health care spending. N Engl J Med. 2012;367:1724-31.

29. Wu B, Zhang Q, Sun J. Cost-effectiveness of nivolumab plus ipilimumab as first-line therapy in advanced renal-cell carcinoma. J Immun Ther Cancer. 2018;6:124.

30. Hoyle M, Green C, Thompson-Coon J, et al. Cost-effectiveness of temsirolimus for first line treatment of advanced renal cell carcinoma. Value Health. 2010;13:61-8.

31. Paz-Ares L, Dvorkin M, Chen Y, et al. Durvalumab plus platinum-etoposide versus platinum-etoposide in first-line treatment of extensive-stage small-cell lung cancer (CASPIAN): a randomised, controlled, open-label, phase 3 trial. Lancet. 2019;394:1929-39.

32. Horn L, Mansfield AS, Szczęsna A, et al. First-line atezolizumab plus chemotherapy in extensive-stage small-cell lung cancer. N Engl J Med. 2018;379:2220-9.

33. Ding $\mathrm{D}, \mathrm{Hu} \mathrm{H}$, Li S, et al. Cost-effectiveness analysis of durvalumab plus chemotherapy in the first-line treatment of extensive-stage small cell lung cancer. J Natl Compr Canc Netw. 2021. https://doi.org/10.6004/ jnccn.2020.7796.

34. Zhou K, Zhou J, Huang J, et al. Cost-effectiveness analysis of atezolizumab plus chemotherapy in the first-line treatment of extensive-stage smallcell lung cancer. Lung Cancer. 2019;130:1-4.

35. Chouaid C, Bensimon L, Clay E, et al. Cost-effectiveness analysis of pembrolizumab versus standard-of-care chemotherapy for first-line treatment of PD-L1 positive (>50\%) metastatic squamous and non-squamous nonsmall cell lung cancer in France. Lung Cancer. 2019;127:44-52.

36. Brody R, Zhang Y, Ballas M, et al. PD-L1 expression in advanced NSCLC: insights into risk stratification and treatment selection from a systematic literature review. Lung Cancer. 2017;112:200-15.

37. Reck M, Rodríguez-Abreu D, Robinson AG, et al. Pembrolizumab versus chemotherapy for PD-L1-positive non-small-cell lung cancer. N Engl J Med. 2016;375:1823-33

38. Tsuruoka K, Horinouchi H, Goto Y, et al. PD-L1 expression in neuroendocrine tumors of the lung. Lung Cancer. 2017;108:115-20.

39. Antonia SJ, López-Martin JA, Bendell J, et al. Nivolumab alone and nivolumab plus ipilimumab in recurrent small-cell lung cancer (CheckMate 032): a multicentre, open-label, phase 1/2 trial. Lancet Oncol. 2016;17:883-95.

40. Ishii H, Azuma K, Kawahara A, et al. Significance of programmed cell death-ligand 1 expression and its association with survival in patients with small cell lung cancer. J Thorac Oncol. 2015;10:426-30.

41. Schultheis AM, Scheel AH, Ozretić L, et al. PD-L1 expression in small cell neuroendocrine carcinomas. Eur J Cancer. 2015;51:421-6.

42. Gandara DR, Paul SM, Kowanetz M, et al. Blood-based tumor mutational burden as a predictor of clinical benefit in non-small-cell lung cancer patients treated with atezolizumab. Nat Med. 2018;24:1441-8.

43. Samstein RM, Lee $\mathrm{C}-\mathrm{H}$, Shoushtari AN, et al. Tumor mutational load predicts survival after immunotherapy across multiple cancer types. Nat Genet. 2019;51:202-6.

44. Herbst RS, Soria J-C, Kowanetz M, et al. Predictive correlates of response to the anti-PD-L1 antibody MPDL3280A in cancer patients. Nature. 2014;515:563-7.

45. She L, Hu H, Liao M, et al. Cost-effectiveness analysis of pembrolizumab versus chemotherapy as first-line treatment in locally advanced or metastatic non-small cell lung cancer with PD-L1 tumor proportion score 1\% or greater. Lung Cancer. 2019;138:88-94.

46. Hu H, She L, Liao M, et al. Cost-effectiveness analysis of nivolumab plus ipilimumab vs. chemotherapy as first-line therapy in advanced non-small cell lung cancer. Front Oncol. 2020;10:1649.

47. Tartari F, Santoni M, Burattini L, et al. Economic sustainability of anti-PD-1 agents nivolumab and pembrolizumab in cancer patients: Recent insights and future challenges. Cancer Treat Rev. 2016;48:20-4.

\section{Publisher's Note}

Springer Nature remains neutral with regard to jurisdictional claims in published maps and institutional affiliations.
Ready to submit your research? Choose BMC and benefit from:

- fast, convenient online submission

- thorough peer review by experienced researchers in your field

- rapid publication on acceptance

- support for research data, including large and complex data types

- gold Open Access which fosters wider collaboration and increased citations

- maximum visibility for your research: over $100 \mathrm{M}$ website views per year

At BMC, research is always in progress.

Learn more biomedcentral.com/submissions 